\title{
Management and outcomes of pregnancy with or without lupus nephritis: a systematic review and meta-analysis
}

This article was published in the following Dove Press journal:

Therapeutics and Clinical Risk Management

\author{
Jiayue $\mathrm{Wu}^{1-3, *}$ \\ Jinghang $\mathrm{Ma}^{\mathrm{I}, 2, *}$ \\ Wei-Hong Zhang ${ }^{3}$ \\ Wen $\mathrm{Di}^{1,2}$ \\ 'Department of Obstetrics and \\ Gynecology, Ren Ji Hospital, School \\ of Medicine, Shanghai Jiao Tong \\ University, Shanghai, People's Republic \\ of China; ${ }^{2}$ Shanghai Key Laboratory \\ of Gynecologic Oncology, Ren Ji \\ Hospital, School of Medicine, Shanghai \\ Jiao Tong University, Shanghai, People's \\ Republic of China; ${ }^{3}$ International \\ Centre for Reproductive Health \\ (ICRH), Ghent University, Ghent, \\ Belgium \\ *These authors contributed equally \\ to this work
}

Background: Although it is well established that systemic lupus erythematosus (SLE) negatively affects pregnancy outcomes, there is insufficient evidence on the effect of lupus nephritis (LN) on antenatal management and pregnancy outcomes. We performed a systematic review and meta-analysis to determine the association of $\mathrm{LN}$ with management and pregnancy outcomes in SLE patients.

Methods: Embase, Medline, Cochrane, and ClinicalTrials.gov were carefully searched for relevant English and Chinese language studies. A total of 2,987 articles were reviewed. Data were extracted that compared management and pregnancy outcomes in SLE pregnant women with LN vs without LN. Risk of bias was assessed by a modified version of the Newcastle-Ottawa Scale and the STROBE checklist. Combined odds ratios (OR) and 95\% confidence intervals (CI) were obtained and sensitivity analysis was performed using RevMan 5.3 software.

Results: Sixteen studies, including 1,760 pregnant patients with SLE, were included. Gestational hypertension $(\mathrm{OR}=5.65,95 \% \mathrm{CI}=2.94-10.84)$, preeclampsia $(\mathrm{OR}=2.84,95 \% \mathrm{CI}=1.87-4.30)$, SLE flare $(\mathrm{OR}=2.66,95 \% \mathrm{CI}=1.51-4.70)$, renal flare $(\mathrm{OR}=15.18,95 \% \mathrm{CI}=5.89-39.14)$, proteinuria $(\mathrm{OR}=8.86,95 \% \mathrm{CI}=4.75-16.52)$, and hypocomplementemia $(\mathrm{OR}=2.86,95 \% \mathrm{CI}=1.68-4.87)$ were significantly affected in pregnant women with LN. Anti-Sjögren's syndrome-related antigen $\mathrm{A} /$ Ro autoantibodies were negatively associated with pregnant women with $\mathrm{LN}(\mathrm{OR}=0.57$, 95\% CI $=0.33-0.98)$. Pregnant women with $\mathrm{LN}$ presented a significant decrease in live births $(\mathrm{OR}=0.62,95 \% \mathrm{CI}=0.49-0.80)$ and a significant increase in preterm births $(\mathrm{OR}=1.92,95 \%$ $\mathrm{CI}=1.49-2.49)$ and fetal growth restriction $(\mathrm{OR}=1.43,95 \% \mathrm{CI}=1.08-1.91)$. Regarding antenatal management, steroids $(\mathrm{OR}=2.48,95 \% \mathrm{CI}=1.59-3.87)$ and immunosuppressant treatment $(\mathrm{OR}=6.77,95 \% \mathrm{CI}=3.30-13.89)$ were more frequently used in women with $\mathrm{LN}$.

Conclusion: This review identified a significant association between the aforementioned outcomes and SLE pregnant patients with LN. In patients with SLE, LN increased the risks for adverse pregnancy outcomes and the use of medication. Therefore, special treatment and close monitoring should be allocated to pregnant women with LN.

Keywords: systemic lupus erythematosus, lupus nephritis, maternal outcomes, fetal outcomes, antenatal management

Department of Obstetrics and Gynecology, Ren Ji Hospital, School of Medicine, Shanghai Jiao Tong University, Shanghai 200127, People's Republic of China

Emaildiwen163@I63.com

Wei-Hong Zhang International Centre for Reproductive Health (ICRH), Ghent University, 9000 Ghent, Belgium Email weihong.zhang@ugent.be

\section{Introduction}

Systemic lupus erythematosus (SLE) is a multisystemic autoimmune disease that primarily affects women of childbearing age. Women with SLE have a normal fertility rate, ${ }^{1}$ making pregnancy a frequent subject of interest in these patients. Pregnancy and SLE are reciprocally adversely affected. Pregnancy may increase SLE activity, and have short or long-term adverse effects on renal function, while SLE can result 
in adverse pregnancy outcomes and complications such as preeclampsia, preterm delivery, and fetal loss. ${ }^{2}$ In past decades, SLE was a contraindication for pregnancy. However, with improvements in treatment and multidisciplinary management, the success rate of delivery in women with SLE has greatly improved. ${ }^{3}$ Women with SLE can have successful pregnancies given that there is optimal timing of conception and close management during pregnancy.

The effects of lupus nephritis (LN) on fetal and maternal outcomes have been extensively studied. Khamashta reported that LN is a risk factor for pregnancy loss, especially in patients with renal impairment. ${ }^{4}$ Moreover, higher incidences of fetal loss, intrauterine growth restriction (IUGR), and gestational hypertension have been reported, even in patients with LN with non-active SLE. ${ }^{5}$ Smyth et al reported that LN was associated with premature birth and hypertension during pregnancy in a systematic review in $2010 .{ }^{6}$ However, these reports had several limitations, such as a retrospective study design, insufficient number of participants, and limited scope of analyzed outcomes.

Therefore, we conducted a systematic review and metaanalysis of management and pregnancy outcome in women with SLE with or without LN to examine the effect and quantifiably clarify the effect of LN on pregnancy outcome, which also serves as an updated systemic review with a more complete analysis of pregnancy outcomes and antenatal management.

\section{Materials and methods Literature search}

We carefully searched Embase, Medline database, ClinicalTrials.gov, and Cochrane Database of Randomized Controlled Trials for relevant English and Chinese language studies on June 28, 2017. In addition, we examined the reference lists of the literature that met the inclusion criteria of our study. The search was conducted in accordance with the Preferred Reporting Items for Systematic Reviews and Meta-Analyses guidelines. ${ }^{7}$ During the process of searching, we used a protocol that included the Cochrane Collaboration's search strategy for randomized controlled trials and related exploded terms, including Medical Subject Headings in combination with keywords and the following terms: systemic lupus erythematosus, SLE, lupus nephritis, pregnancy, pregnancy outcomes, maternal outcomes, fetal outcomes, and gynecology.

The search was carried out in June 2017, and only included studies that were published since the year 2000 . Studies published prior to the year 2000 were excluded from this analysis because of more recent changes in health care facilities, medical conditions, laboratory data, and diagnostic criteria, and would therefore introduce bias to the analysis. The full results of our search strategy are shown in Appendix 1 Tables S1-S4.

\section{Study selection}

Studies were included if:

1. They compared pregnant women with SLE with LN (the experimental group) versus pregnant women with SLE without LN (non-LN) (the control group).

2. They reported adverse maternal and/or fetal outcomes. Studies were excluded if:

1. They did not compare pregnant women with $\mathrm{LN}$ versus pregnant women with SLE without LN (only included the experimental group without including the control group).

2. They compared pregnant women with SLE versus pregnant women without SLE (not the objective of the present study).

3. They were reviews, editorials, guidelines, case reports, letters, abstracts, or summaries of meetings.

4. They did not involve pregnant women.

5. They did not report adverse maternal and/or fetal outcomes.

6. They were duplicates of the same studies.

Two authors (JY W and JH M) independently performed the first selection of studies based on titles and abstracts, and then examined the selected full texts. Disagreements between reviewers were resolved by consensus. If no agreement could be reached, the matter was resolved through discussion with another author (WH Z).

\section{Analysis of outcomes}

The following information was extracted from each included study:

- Names of authors

- Publication year

- Period of participants' enrollment

- Geographical region of the study

- Type of study

- Types and total number of participants in each group

- Number of events in each group

- Baseline characteristics of the participants

- Diagnostic criteria of SLE, criteria for the histological diagnosis of LN, SLE activity score, and diagnostic criteria of flares

- Outcomes of interest 
The following outcomes were defined as follows:

\section{Maternal outcomes}

1. Spontaneous abortion: spontaneous termination of pregnancy prior to 20 weeks of gestation, or prior to 24 weeks of gestation.

2. Gestational hypertension: the presence of blood pressure $\geq 140 / 90 \mathrm{mmHg}$ on at least two occasions $\geq 6 \mathrm{~h}$ apart, arising de novo after the 20th week of pregnancy.

3. Preeclampsia: pregnancy-induced hypertension with proteinuria $>0.3 \mathrm{~g} / \mathrm{L} / \mathrm{d}$ in the absence of urinary tract infection, or abrupt onset of hypertension and proteinuria after 20 weeks of gestation.

4. SLE flare: considered as: 1) new signs of active disease by clinical and laboratory variables or change in therapy; 2) change in Systemic Lupus Erythematosus Disease Activity Index 2000 (SLEDAI-2k) score ${ }^{8}$ 3) change in Systemic Lupus Erythematosus Disease Activity Index (SLEDAI) score; ${ }^{9}$ 4) change in Lupus Activity Index in Pregnancy; ${ }^{10} 5$ ) change in the physician global assessment. $^{11}$

5. Renal flare: considered as worsening proteinuria (defined as an increase by $2 \mathrm{~g} / 24 \mathrm{~h}$ if baseline proteinuria was $<3.5 \mathrm{~g} / 24 \mathrm{~h}$, or doubling of proteinuria in women with previous nephrotic range proteinuria), urinary casts, dysmorphic hematuria, reduced levels of $\mathrm{C} 3$ and/or $\mathrm{C} 4$, or rise in serum creatinine of $>30 \%$. $^{12,13}$

6. Cesarean section: included all of the operative indications.

7. Premature rupture of membranes (PROM): diagnosed as a rupture of the amniotic sac prior to the onset of labour.

8. Gestational diabetes mellitus (GDM): defined as any degree of glucose intolerance with onset or first recognition during pregnancy. The diagnosis of GDM is made if there is at least one abnormal value $(\geq 5.1 \mathrm{mmol} / \mathrm{L}$, $10.0 \mathrm{mmol} / \mathrm{L}, 8.5 \mathrm{mmol} / \mathrm{L}$ for fasting, $1-\mathrm{h}$, and $2-\mathrm{h}$ plasma glucose concentration, respectively) after a 75 g oral glucose tolerance test undertaken at 26-28 weeks gestation.

9. Antiphospholipid antibodies (aPL) and antiphospholipid syndrome (APS): aPL included lupus anticoagulant, anticardiolipin antibodies, and $\beta 2$-glycoprotein I; APS was defined according to the Sapporo criteria. ${ }^{14}$

10. Active disease at conception: defined according to the criteria of each study.

11. Proteinuria during pregnancy: proteinuria $\geq 300 \mathrm{mg} / 24 \mathrm{~h}$.
12. Positivity for anti-Sjögren's syndrome-related antigen $A$ (anti-SSA)/Ro autoantibodies.

13. Hypocomplementemia: defined as low C3 and/or C4 levels.

\section{Fetal outcomes}

1. Live birth: the birth of a living child.

2. Stillbirth or intrauterine fetal death: spontaneous termination of pregnancy after 20 weeks of gestation or after 24 weeks of gestation.

3. Preterm birth: delivery $<37$ weeks of gestation.

4. Low birth weight (LBW)/IUGR/small for gestational age (SGA): neonatal birth weight $<2,500 \mathrm{~g}$, or an estimated birth weight of less than the lower $10 \%$ limit of the CI of the normal curve for gestation weight.

5. Neonatal lupus: included all types of neonatal lupus, as well as either cutaneous lesions and/or congenital heart block.

6. Congenital malformation: included all types of congenital malformation.

7. One-min Apgar scores: method for quickly evaluating the health of a neonate.

\section{Antenatal management (management of medication use during pregnancy)}

It included all forms of the following drugs, regardless of dosage and method of administration:

- Steroids

- Antimalarials

- Immunosuppressants

- Aspirin

\section{Data collection}

A standardized data extraction sheet was developed to record data. One author (JY W) extracted the aforementioned data from the included studies, and the other author (JH M) checked the extracted data. Disagreements were resolved by discussion between the two authors. If no agreement could be reached, another author (WH Z) would resolve the matter.

\section{Assessment of risk of bias}

The quality of the studies was reviewed by JY W and JH M using a modified version of the Newcastle-Ottawa Scale (NOS) for cohort studies and cross-sectional studies. ${ }^{15,16}$ Disagreements were resolved by consensus. This scale assesses risk of bias in the following three parameters: selection, comparability, and exposure/outcome. The total scores were 9 for cohort studies and 8 for cross-sectional 
studies. A score $\geq 7$ in cohort studies and $\geq 6$ in crosssectional studies were indicative of a high quality study. ${ }^{17}$ Furthermore, each study was assessed using the Strengthening the Reporting of Observational Studies in Epidemiology (STROBE) checklist, which is a 22-item checklist that assesses the essential items of observational studies. ${ }^{18}$ The purpose of STROBE is not to assess quality, but to ensure clear presentation of the study. The results of the full-modified versions of the NOS and STROBE checklists are shown in Appendix 2 Tables S5 and $\underline{\text { S6}}$ and Appendix 3 Table S7. Funnel plots were used to visually estimate publication bias.

\section{Statistical analysis}

Dichotomous data were used for comparisons. The metaanalyses were performed by calculating odds ratios (OR) using a fixed- or random-effects model according to their heterogeneity. The heterogeneity across studies was assessed by the $Q$-statistic and $I^{2}$ statistic tests. When $P_{Q}<0.1$ or $I^{2}>50 \%$, the heterogeneity was considered significant. Therefore, a random-effects model was used to calculate the pooled OR, and the sensitivity test was conducted to exclude studies that increased the heterogeneity. Otherwise, the fixed-effects model was used. Outcomes that were included in less than three studies or that were defined differently were evaluated qualitatively rather than by meta-analysis. All tests were twotailed, and $P<0.05$ was considered statistically significant. Analyses were carried out using RevMan 5.3 software.

\section{Results}

\section{Study selection}

A flow diagram of the study selection process is shown in Figure 1. A total of 2,987 articles were extracted from the aforementioned databases. Following the screening of titles and abstracts, 104 full-text articles were assessed for eligibility. Finally, 16 articles were included in the quantitative synthesis of this systematic review.

\section{Study characteristics}

The main characteristics of the included studies are shown in Table 1. There were 15 retrospective studies and one cross-sectional study. Patients were enrolled between the years 1970 and 2015, and studies were conducted in different regions such as Europe, South Africa, Canada, the US, and some Asian countries.

The 16 studies included a total of 665 LN pregnancies (experimental group) and 1,095 non-LN pregnancies (control group). The maternal age at delivery was reported in all studies, and all participants were of childbearing age. However, some studies only reported the mean age of patients

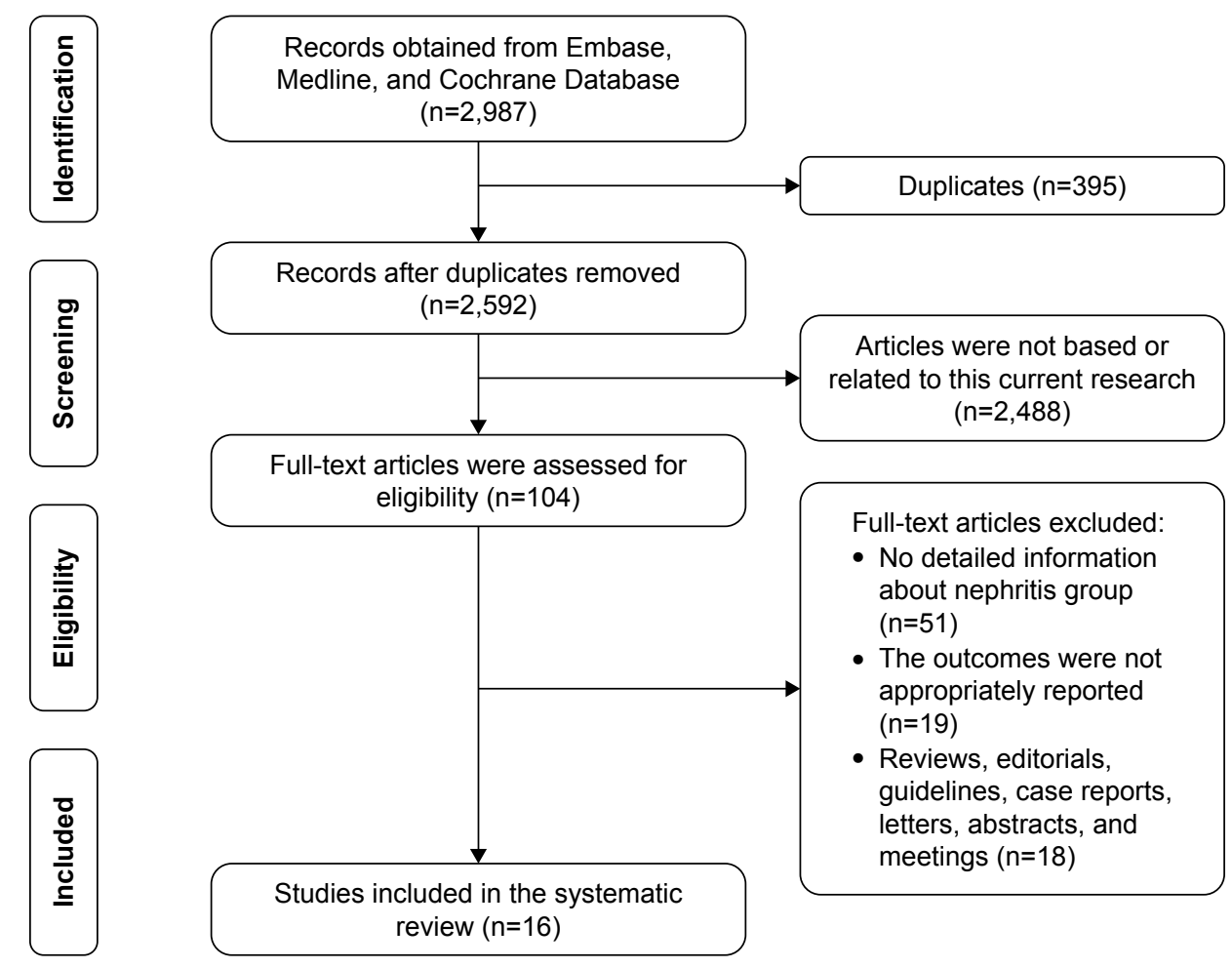

Figure I Flow diagram demonstrating the study selection process. 


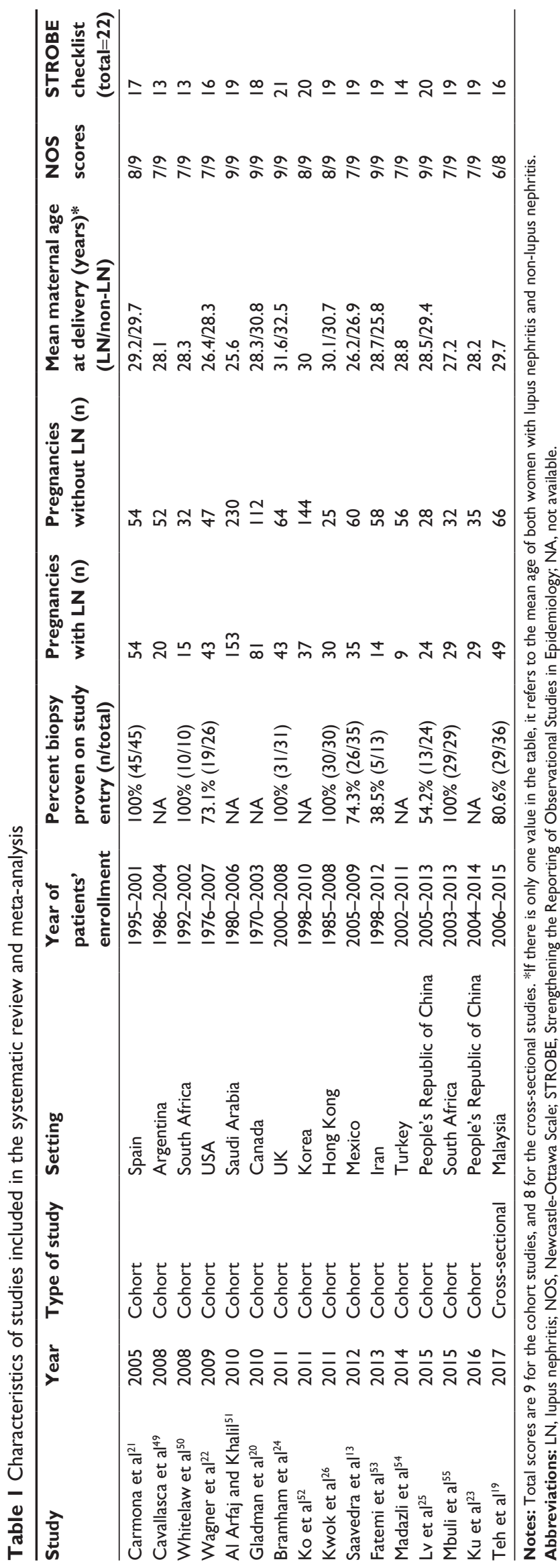

in both group. Additionally, not all participants in each study had biopsy-proven LN. Some studies exclusively included patients with biopsy-proven LN $(n=5)$ as the experimental group, while others had varying percentages of patients with biopsy-proven $\mathrm{LN}(\mathrm{n}=5)$. However, all participants were confirmed to have been diagnosed with SLE and LN upon enrollment in each study.

The diagnostic criteria for SLE varied in each study. The 1997 American College of Rheumatology criteria were the mostly widely used (12/16). Others used the older version, while one did not indicate which criteria were used. The definitions of disease activity also varied. The majority (7/16) used the SLE disease activity index (SLEDAI or SLEDAI-2K), while six studies defined disease activity according to organ involvement and laboratory abnormalities. LN associated with SLE was determined primarily according to the 1995 classification system of renal biopsies of the World Health Organization (7/16), while others also used clinical and laboratory records. The definition of SLE flare during pregnancy varied as well. Most studies (13/16) defined it as new signs of active disease by clinical and laboratory variables or change in therapy (Table 2).

\section{Risk of bias within studies}

The risk of bias within the studies was assessed by a modified version of the NOS for cohort studies and cross-sectional studies, and a STROBE checklist. The NOS and STROBE scores from each study are shown in Table 1. The total NOS scores were 9 for the cohort studies and 8 for the crosssectional studies. The STROBE checklist has a total score of 22. All included studies were of high quality.

\section{Maternal outcomes}

We analyzed 13 maternal outcomes. Among them, 10 were included in the quantitative synthesis, and three were analyzed qualitatively. The outcomes are shown in Appendix 4 Table S8.

\section{Quantitative synthesis and heterogeneity analyses}

In the present analysis, maternal outcomes in pregnant women with SLE with LN were compared with maternal outcomes in pregnant women with SLE without LN. Pregnant women with LN showed a significant association with gestational hypertension $(\mathrm{OR}=5.65,95 \% \mathrm{CI}=2.94-10.84)$, preeclampsia $(\mathrm{OR}=2.84,95 \% \mathrm{CI}=1.87-4.30)$, renal flare $(\mathrm{OR}=15.18,95 \% \mathrm{CI}=5.89-39.14)$, proteinuria $(\mathrm{OR}=8.86$, $95 \% \mathrm{CI}=4.75-16.52)$, and hypocomplementemia $(\mathrm{OR}=2.86$, 
Table 2 Outcomes reported, SLE diagnostic criteria, lupus nephritis diagnostic criteria, disease activity rates, and flare definition upon study enrollment in the studies included for analysis

\begin{tabular}{|c|c|c|c|c|c|c|}
\hline Study & Maternal outcomes & Fetal outcomes & $\begin{array}{l}\text { SLE } \\
\text { diagnostic } \\
\text { criteria }\end{array}$ & $\begin{array}{l}\text { LN } \\
\text { diagnosis } \\
\text { criteria }\end{array}$ & $\begin{array}{l}\text { Disease } \\
\text { activity }\end{array}$ & Flare \\
\hline Carmona et $\mathrm{al}^{21}$ & $\begin{array}{l}\text { Spontaneous abortion, gestational } \\
\text { hypertension, preeclampsia, cesarean } \\
\text { section, flare, renal flare, aPL positive, } \\
\text { active disease at conception }\end{array}$ & $\begin{array}{l}\text { Fetal loss, stillbirth, premature } \\
\text { birth, live birth }\end{array}$ & 1 & 1 & 4 & 1 \\
\hline Cavallasca et a ${ }^{49}$ & $\begin{array}{l}\text { Spontaneous abortion, gestational } \\
\text { hypertension, preeclampsia }\end{array}$ & $\begin{array}{l}\text { Stillbirth, premature birth, full-term } \\
\text { birth, LBW }\end{array}$ & 2 & NA & I & 1 \\
\hline Whitelaw et a ${ }^{50}$ & Spontaneous abortion & $\begin{array}{l}\text { Premature birth, full-term birth, } \\
\text { IUGR, live birth }\end{array}$ & 1 & 1 & 3 & 1 \\
\hline Wagner et $\mathrm{a}^{22}$ & $\begin{array}{l}\text { Spontaneous abortion, gestational } \\
\text { hypertension, preeclampsia, } \\
\text { cesarean section }\end{array}$ & $\begin{array}{l}\text { Premature birth, full-term birth, } \\
\text { SGA, fetal loss, stillbirth, neonatal } \\
\text { death, neonatal lupus }\end{array}$ & 1 & I & I & 1 \\
\hline Al Arfaj and Khalii ${ }^{51}$ & Miscarriages & $\begin{array}{l}\text { Premature birth, full-term birth, } \\
\text { stillbirth, IUGR, live birth }\end{array}$ & 2 & NA & NA & 1 \\
\hline Gladman et $\mathrm{al}^{20}$ & $\begin{array}{l}\text { Spontaneous abortion, gestational } \\
\text { hypertension, preeclampsia, GDM, } \\
\text { medicine, aPL positive, SLE flare }\end{array}$ & $\begin{array}{l}\text { Live birth, stillbirth, LBW, } \\
\text { congenital malformation, neonatal } \\
\text { lupus }\end{array}$ & NA & NA & 2,6 & 2 \\
\hline Bramham et a ${ }^{24}$ & $\begin{array}{l}\text { Preeclampsia, cesarean section, SLE flare, } \\
\text { renal flare, aPL positive, APS, medication }\end{array}$ & $\begin{array}{l}\text { Live birth, intrauterine death, SGA, } \\
\text { I-min Apgar }<7\end{array}$ & I & I & I & 1 \\
\hline Ko et $\mathrm{a}^{52}$ & Miscarriages & $\begin{array}{l}\text { Stillbirth, neonatal death, live birth, } \\
\text { preterm birth, full-term birth, IUGR }\end{array}$ & 1 & NA & 3 & 1 \\
\hline Kwok et $\mathrm{al}^{26}$ & $\begin{array}{l}\text { Spontaneous abortion, SLE flares, } \\
\text { preeclampsia, APS, medications }\end{array}$ & $\begin{array}{l}\text { Stillbirth, fetal loss, SGA, IUGR, } \\
\text { preterm birth }\end{array}$ & 2 & I & 3,5 & 1 \\
\hline Saavedra et $\mathrm{al}^{13}$ & $\begin{array}{l}\text { Spontaneous abortion, cesarean section, } \\
\text { active SLE at conception, SLE flares, } \\
\text { PROM, renal flare, preeclampsia, APS, } \\
\text { proteinuria, medication }\end{array}$ & $\begin{array}{l}\text { Stillbirth fetal loss, neonatal death, } \\
\text { live birth, LBW, preterm birth }\end{array}$ & I, 2 & I, 3 & I & 1 \\
\hline Fatemi et $\mathrm{al}^{53}$ & $\begin{array}{l}\text { Abortion, SLE flare, preeclampsia, APS, } \\
\text { low C3, C4, medication }\end{array}$ & Stillbirth, premature birth & 1 & 2 & 2 & 3 \\
\hline Madazli et $\mathrm{al}^{54}$ & IUGR and/or preeclampsia & $\begin{array}{l}\text { Fetal loss, premature birth, IUGR } \\
\text { and/or preeclampsia }\end{array}$ & 1 & NA & I & 1 \\
\hline Lv et $\mathrm{al}^{25}$ & $\begin{array}{l}\text { Gestational hypertension, preeclampsia, } \\
\text { flare, renal damage, low } \mathrm{C} 3 \text {, low } \mathrm{C} 4, \mathrm{acL} \\
\text { and } \beta 2 \mathrm{GPI} \text { positive, proteinuria }\end{array}$ & $\begin{array}{l}\text { Live births, gestational age, SGA, } \\
\text { fetal loss, fetal malformation, I-min } \\
\text { Apgar scores }\end{array}$ & 1 & NA & 7 & 4 \\
\hline Mbuli et a ${ }^{55}$ & $\begin{array}{l}\text { Miscarriage, cesarean section, flare, } \\
\text { preeclampsia }\end{array}$ & $\begin{array}{l}\text { Stillbirth, premature birth, full-term } \\
\text { birth, live birth }\end{array}$ & 1 & NA & I & 1 \\
\hline Ku et $\mathrm{a}^{23}$ & $\begin{array}{l}\text { SLE flare, proteinuria, } \\
\text { hypocomplementemia, disease activity, } \\
\text { gestational hypertension, proteinuria }\end{array}$ & $\begin{array}{l}\text { Fetal loss, premature birth, IUGR, } \\
\text { neonatal lupus, neonatal heart } \\
\text { disease, I-min Apgar scores }\end{array}$ & I & NA & 2 & 1 \\
\hline Teh et al ${ }^{19}$ & $\begin{array}{l}\text { SLE flare, aPL positive, hypertension, } \\
\text { preeclampsia, PROM, eclampsia, GDM }\end{array}$ & $\begin{array}{l}\text { Premature birth, IUGR, fetal loss, } \\
\text { live births }\end{array}$ & I & I & 3,8 & I, 3,5 \\
\hline
\end{tabular}

Notes: Diagnostic criteria: I) ACR 1997 criteria; ${ }^{56}$ 2) ACR 1982 criteria. ${ }^{57}$ Histological lupus nephritis: I) The 1995 World Health Organization categorization for lupus nephritis; ${ }^{58}$ 2) the 2004 classification of the International Society of Nephrology/Renal Pathology Society; ${ }^{59}$ 3) nephritis was established clinical and biochemically. Activity: I) Organ involvement and laboratory abnormalities; 2) SLEDAI-2k; 3) SLEDAI; 4) lupus activity criteria count; 5) Systemic Lupus Erythematosus Pregnancy Disease Activity Index; 6) Adjusted Mean Systemic Lupus Erythematosus Disease Activity Index; 7) LAI-P; 8) PGA. Flare: I) New signs of active disease by clinical and laboratory variables or change in therapy; 2) SLEDAI-2k; 3) SLEDAI score; 4) change in LAI-P; 5) change in PGA. aPL include lupus anticoagulant, anticardiolipin antibodies, and $\beta 2$-glycoprotein I. APS was defined according to the Sapporo criteria.

Abbreviations: SLE, systemic lupus erythematosus; LN, lupus nephritis; aPL, antiphospholipid antibodies; LBW, low birth weight; NA, not available; IUGR, intrauterine growth restriction; SGA, small for gestational age; GDM, gestational diabetes mellitus; APS, antiphospholipid syndrome; PROM, premature rupture of membranes; $\beta 2$ GPI, 22-glycoprotein; ACR, American College of Rheumatology; SLEDAI-2k, Systemic Lupus Erythematosus Disease Activity Index 2000; SLEDAI, Systemic Lupus Erythematosus Disease Activity Index; LAI-P, Lupus Activity Index in Pregnancy; PGA, Physician Global Assessment.

$95 \% \mathrm{CI}=1.68-4.87$ ), with low statistical heterogeneity in all outcomes, and the fixed-effects model was used. SLE flare was also significantly affected in pregnant women with LN $(\mathrm{OR}=2.66,95 \% \mathrm{CI}=1.51-4.70)$, with statistical heterogeneity across studies $\left(I^{2}=69 \%, P_{Q}=0.001\right)$, and the random-effects model was used.

There was less positivity for anti-SSA/Ro autoantibodies in pregnant women with SLE with LN compared with those 
without $\mathrm{LN}(\mathrm{OR}=0.57,95 \% \mathrm{CI}=0.33-0.98)$, with statistical heterogeneity across studies $\left(I^{2}=43 \%, P_{Q}=0.15\right)$, and the fixed-effects model was used.

However, spontaneous abortion (which was divided into two subgroups according to different outcome definitions) $(\mathrm{OR}=1.25,95 \% \mathrm{CI}=0.92-1.70)$, cesarean section $(\mathrm{OR}=1.17$, $95 \% \mathrm{CI}=0.79-1.74)$, and aPL positivity and APS (OR=0.98, 95\% CI $=0.71-1.35$ ) were not significantly different between pregnant women with $\mathrm{LN}$ and those without LN. The results of maternal outcomes are shown in Figure 2 and Table 3.

\section{Sensitivity analyses}

Regarding SLE flare, the statistical heterogeneity was high $\left(I^{2}=69 \%, P_{Q}=0.001\right)$. Therefore, sensitivity analyses were performed (Table 4A). The sensitivity analyses showed that after omitting the studies by Bramham et al or Lv et al, SLE flare was still significantly affected in pregnant women with LN $\left(\mathrm{OR}=3.12,95 \% \mathrm{CI}=1.83-5.32, I^{2}=59 \%, P_{Q}=0.02\right.$; $\mathrm{OR}=2.22,95 \% \mathrm{CI}=1.34-3.68, I^{2}=59 \%, P_{Q}=0.02$, respectively). These data indicated that the pooled results remained robust in the SLE flare outcome.

\begin{tabular}{|c|c|c|c|c|c|c|c|c|}
\hline & & & & & pontan & eous abortion & & \\
\hline Study & $\begin{array}{l}\text { LN } \\
\text { Events }\end{array}$ & Total & $\begin{array}{l}\text { Non-LN } \\
\text { Events }\end{array}$ & Total & $\begin{array}{l}\text { Weight } \\
(\%)\end{array}$ & $\begin{array}{l}\text { Odds ratio } \mathrm{M}-\mathrm{H} \text {, } \\
\text { fixed, } 95 \% \mathrm{Cl}\end{array}$ & Year & $\begin{array}{l}\text { Odds ratio } \mathrm{M}-\mathrm{H} \text {, } \\
\text { fixed, } 95 \% \mathrm{Cl}\end{array}$ \\
\hline Spontaneous abortio & 20 weeks & & & & & & & \\
\hline Carmona et $\mathrm{al}^{21}$ & 8 & 54 & 9 & 54 & 10.6 & $0.87(0.31,2.45)$ & 2005 & $\rightarrow$ \\
\hline Whitelaw et al ${ }^{50}$ & 3 & 15 & 5 & 32 & 3.5 & $1.35(0.28,6.58)$ & 2008 & \\
\hline Gladman et $\mathrm{al}^{20}$ & 18 & 81 & 24 & 112 & 21.7 & $1.05(0.52,2.09)$ & 2010 & \\
\hline Saavedra et al ${ }^{13}$ & 4 & 35 & 4 & 60 & 3.6 & $1.81(0.42,7.73)$ & 2012 & \\
\hline Subtotal $(95 \% \mathrm{Cl})$ & & 185 & & 258 & 39.4 & $1.10(0.66,1.82)$ & & \\
\hline Total events & 33 & & 42 & & & & & \\
\hline Heterogeneity: $\chi^{2}=0.73$ & $d f=3(P=c$ & $87) ; I^{2}=$ & & & & & & \\
\hline Test for overall effect: $Z$ & $=0.36(P=$ & $72)$ & & & & & & \\
\hline Spontaneous abortio & 24 weeks & & & & & & & \\
\hline Al Arfaj and Khalil et ${ }^{51}$ & 44 & 153 & 50 & 230 & 39.4 & $1.45(0.91,2.32)$ & 2010 & \\
\hline Ko et $\left.a\right|^{52}$ & 4 & 37 & 13 & 144 & 6.6 & $1.22(0.37,3.99)$ & 2011 & \\
\hline Kwok et $\mathrm{al}^{26}$ & 1 & 30 & 0 & 25 & 0.7 & $2.59(0.10,66.49)$ & 2011 & \\
\hline Fatemi et $\mathrm{al}^{53}$ & 5 & 14 & 16 & 58 & 5.5 & $1.46(0.42,5.02)$ & 2013 & \\
\hline Mbuli et al ${ }^{55}$ & 6 & 29 & 8 & 32 & 8.3 & $0.78(0.23,2.61)$ & 2015 & \\
\hline Subtotal $(95 \% \mathrm{Cl})$ & & 263 & & 489 & 60.6 & $1.35(0.92,1.98)$ & & \\
\hline Total events & 60 & & 87 & & & & & \\
\hline Heterogeneity: $\chi^{2}=1.08$ & $d f=4(P=0$ & $90) ; I^{2}=$ & & & & & & \\
\hline Test for overall effect: $z$ & $=1.52(P=0$ & 13) & & & & & & \\
\hline Total $(95 \% \mathrm{Cl})$ & & 448 & & 747 & 100 & $1.25(0.92,1.70)$ & & \\
\hline Total events & 93 & & 129 & & & & & \\
\hline Heterogeneity: $\chi^{2}=2.21$ & $d f=8(P=0$ & $97) ; I^{2}=$ & $\%$ & & & & & \\
\hline $\begin{array}{l}\text { Test for overall effect: } \\
\text { Test for subgroup differ }\end{array}$ & $\begin{array}{l}=1.42(P=0 \\
\text { nces: } \chi^{2}=0\end{array}$ & 15) $d f$ & $(P=0.52$ & $2=0 \%$ & & & & \\
\hline
\end{tabular}

Gestational hypertension

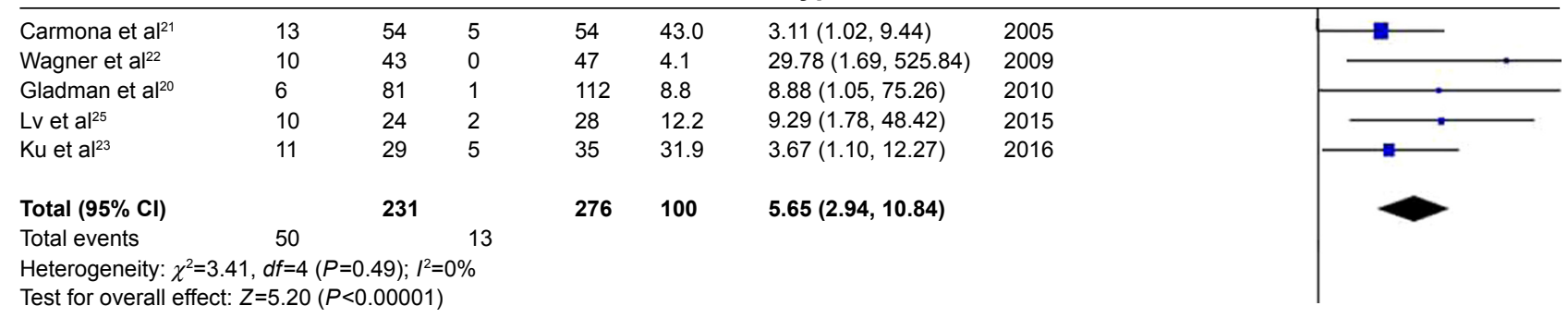

Preeclampsia

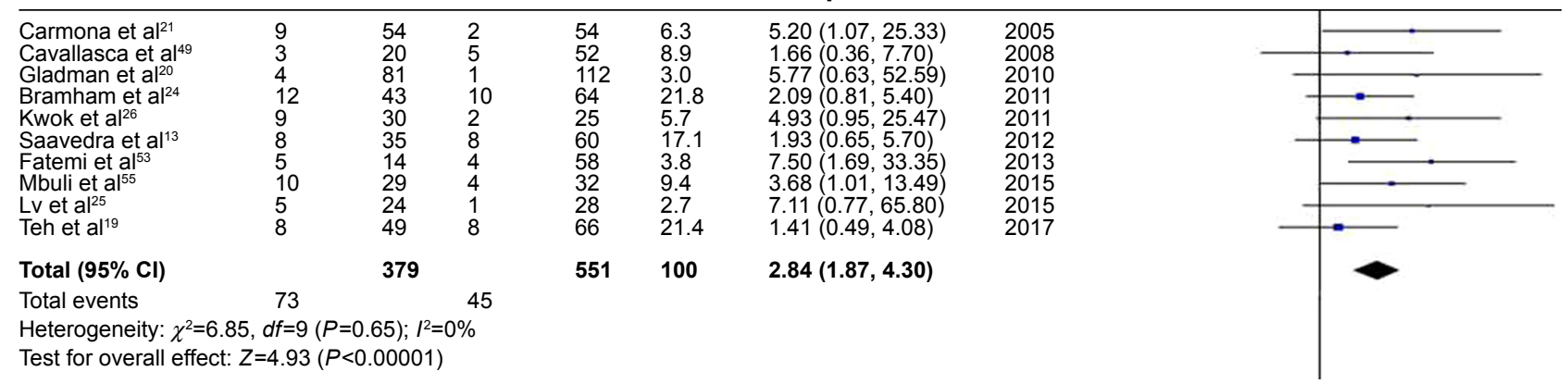

Figure 2 (Continued) 


\section{Hypocomplementemia}

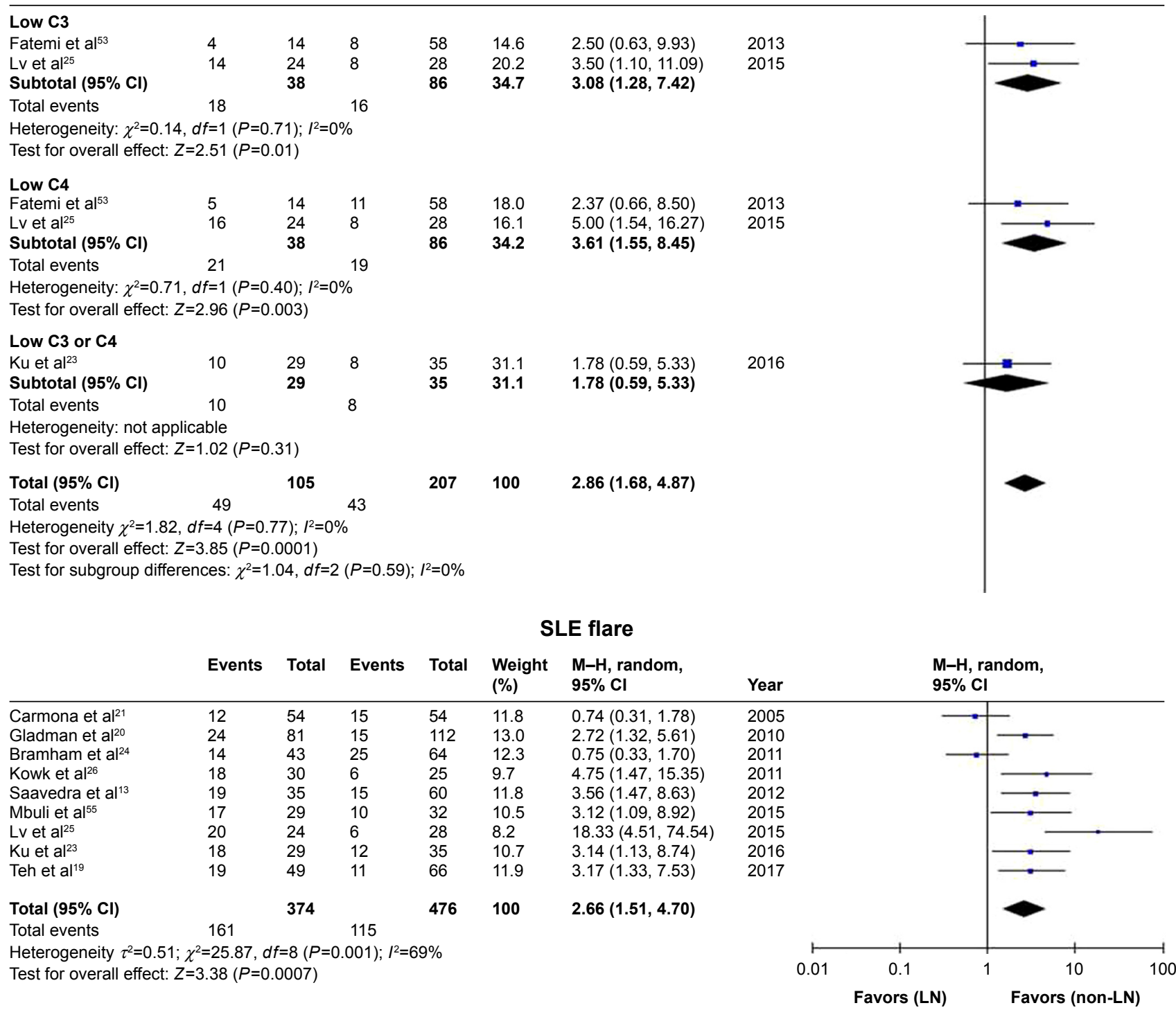

Figure 2 Maternal outcomes observed in pregnant women with SLE with lupus nephritis versus those without lupus nephritis.

Abbreviations: SLE, systemic lupus erythematosus; LN, lupus nephritis; aPL+, antiphospholipid antibodies positive; APS, antiphospholipid syndrome; anti-SSA/Ro+, antiSjögren's syndrome-related antigen A/Ro autoantibodies positive.

\section{Qualitative analyses}

There were two articles that studied PROM, ${ }^{13,19}$ both of which showed no significant difference between pregnant women with LN and those without LN. The same result was found regarding GDM. ${ }^{19,20}$ Saavedra et al found that more pregnancies with $\mathrm{LN}$ had evidence of active disease at conception $(25.7 \%$ vs $6.6 \%, P=0.009)$, and active disease at conception was a predictor for any type of maternal complication $(\mathrm{OR}=16.4,95 \% \mathrm{CI}=1.97-137.2, P=0.01){ }^{13}$ Carmona et al did not identify any differences between the two groups. ${ }^{21}$ The results are shown in Appendix 5 Table S11A.

\section{Fetal outcomes}

We analyzed seven fetal outcomes. Among them, four were included in the quantitative synthesis, and three were qualitative analyses. The outcomes are shown in Appendix 4 Table S9.

\section{Quantitative synthesis and heterogeneity analyses}

In these analyses, fetal outcomes in pregnant women with SLE with LN were compared with fetal outcomes in pregnant women with SLE without LN. Pregnant women with LN showed a significant decrease in live births $(\mathrm{OR}=0.62$, $95 \% \mathrm{CI}=0.49-0.80$ ), with statistical heterogeneity across 
Table 3 Results of meta-analysis

\begin{tabular}{|c|c|c|c|}
\hline $\begin{array}{l}\text { Outcomes } \\
\text { analyzed }\end{array}$ & $\begin{array}{l}\text { No. of } \\
\text { studies } \\
\text { involved (n) }\end{array}$ & $\begin{array}{l}\text { OR with } \\
95 \% \mathrm{Cl}\end{array}$ & $I^{2}$ \\
\hline \multicolumn{4}{|l|}{ Maternal outcomes } \\
\hline Spontaneous abortion & 9 & $1.25(0.92,1.70)$ & $0 \%$ \\
\hline \multicolumn{3}{|l|}{ hypertension } & $0 \%$ \\
\hline Preeclampsia & 10 & $2.84(1.87,4.30)$ & $0 \%$ \\
\hline SLE flare & 9 & $2.66(1.51,4.70)$ & $69 \%$ \\
\hline Renal flare & 4 & $15.18(5.89,39.14)$ & $0 \%$ \\
\hline Cesarean section & 5 & $1.17(0.79,1.74)$ & $38 \%$ \\
\hline aPL and APS & 8 & $0.98(0.7 \mathrm{I}, \mathrm{I} .35)$ & $0 \%$ \\
\hline Proteinuria & 3 & $8.86(4.75,16.52)$ & $0 \%$ \\
\hline SSA+ & 4 & $0.57(0.33,0.98)$ & $43 \%$ \\
\hline Hypocomplementemia & 3 & $2.86(1.68,4.87)$ & $0 \%$ \\
\hline \multicolumn{4}{|l|}{ Fetal outcomes } \\
\hline Live birth & 15 & $0.62(0.49,0.80)$ & $0 \%$ \\
\hline Stillbirth & 9 & $1.68(0.95,2.98)$ & $0 \%$ \\
\hline Preterm birth & 13 & $1.92(1.49,2.49)$ & $0 \%$ \\
\hline LBW/IUGR/SGA & II & $1.43(1.08,1.91)$ & $0 \%$ \\
\hline \multicolumn{4}{|c|}{ Antenatal management } \\
\hline Steroids & 5 & $2.48(1.59,3.87)$ & $27 \%$ \\
\hline Antimalarials & 5 & $0.67(0.30,1.49)$ & $72 \%$ \\
\hline Immunosuppressives & 5 & $6.77(3.30,13.89)$ & $53 \%$ \\
\hline
\end{tabular}

Abbreviations: OR, odds ratio; SLE, systemic lupus erythematosus; aPL, antiphospholipid antibodies; APS, antiphospholipid syndrome; SSA+, Sjögren's syndrome-related antigen A positive LBW, low birth weight; IUGR, intrauterine growth restriction; SGA, small for gestational age.

Table 4 Sensitivity analyses

\begin{tabular}{|c|c|c|c|c|}
\hline \multicolumn{5}{|l|}{ A } \\
\hline Study omitted & $P_{Q}$ & $1^{2}$ & OR & $95 \% \mathrm{Cl}$ \\
\hline Carmona et $\mathrm{al}^{21}$ & 0.01 & $61 \%$ & 3.11 & $1.80,5.37$ \\
\hline Gladman et $\mathrm{a}^{20}$ & 0.0006 & $73 \%$ & 2.69 & I. $38,5.24$ \\
\hline Bramham et $\mathrm{a}^{24}$ & 0.02 & $59 \%$ & 3.12 & $1.83,5.32$ \\
\hline Kwok et a ${ }^{26}$ & 0.001 & $71 \%$ & 2.51 & $1.36,4.62$ \\
\hline Saavedra et al ${ }^{13}$ & 0.0008 & $72 \%$ & 2.58 & I.36, 4.89 \\
\hline Lv et $\mathrm{a}^{25}$ & 0.02 & $59 \%$ & 2.22 & $1.34,3.68$ \\
\hline Mbuli et a ${ }^{55}$ & 0.0006 & $73 \%$ & 2.63 & $1.40,4.96$ \\
\hline $\mathrm{Ku}$ et $\mathrm{a}^{23}$ & 0.0006 & $73 \%$ & 2.63 & $1.40,4.96$ \\
\hline Teh et $\mathrm{al}^{19}$ & 0.0007 & $72 \%$ & 2.63 & $1.38,5.01$ \\
\hline \multicolumn{5}{|l|}{ B } \\
\hline Gladman et $\mathrm{a}^{20}$ & 0.002 & $79 \%$ & 0.66 & $0.20,2.12$ \\
\hline Bramham et $\mathrm{a}^{24}$ & 0.002 & $79 \%$ & 0.64 & $0.21,1.95$ \\
\hline Kwok et a ${ }^{26}$ & 0.25 & $28 \%$ & 0.89 & $0.54,1.46$ \\
\hline Saavedra et $\mathrm{al}^{13}$ & 0.004 & $78 \%$ & 0.59 & $0.21,1.62$ \\
\hline Fatemi et al ${ }^{53}$ & 0.02 & $69 \%$ & 0.52 & $0.23,1.16$ \\
\hline \multicolumn{5}{|l|}{ C } \\
\hline Bramham et $\mathrm{al}^{24}$ & 0.28 & $20 \%$ & 4.66 & $2.04,10.66$ \\
\hline Kwok et a ${ }^{26}$ & 0.005 & $81 \%$ & 7.34 & $1.63,33.09$ \\
\hline Saavedra et al ${ }^{13}$ & 0.007 & $80 \%$ & 7.8 & I. $34,45.38$ \\
\hline Fatemi et $\mathrm{al}^{53}$ & 0.08 & $61 \%$ & 11.64 & $3.94,34.38$ \\
\hline
\end{tabular}

Notes: (A) Outcome: SLE flare. (B) Outcome: antimalarials. (C) Outcome: immunosuppressant-azathioprine.

Abbreviations: SLE, systemic lupus erythematosus; OR, odds ratio. studies $\left(I^{2}=0 \%, P_{Q}=0.53\right)$, and the fixed-effects model was used. In addition, preterm births were significantly higher in neonates born from pregnant women with LN $(\mathrm{OR}=1.92,95 \% \mathrm{CI}=1.49-2.49)$, with low heterogeneity $\left(I^{2}=0 \%, P_{Q}=0.76\right)$, and the fixed-effects model was used. There were significantly more neonates who were classified with LBW/IUGR/SGA in pregnant women with LN (OR=1.43, 95\% CI=1.08-1.91), with low heterogeneity $\left(I^{2}=0 \%, P_{Q}=0.76\right)$, and the fixed-effects model was used.

However, stillbirths (divided into two subgroups according to different outcome definitions, 20 weeks and 24 weeks) were not significantly different between pregnant women with $\mathrm{LN}$ and those without $\mathrm{LN}$, either in subgroups or in total $(\mathrm{OR}=1.25,95 \% \mathrm{CI}=0.92-1.70)$. The results of fetal outcomes are shown in Figure 3 and Table 3.

\section{Qualitative analyses}

A total of six cases of neonatal lupus were identified, and all the included studies showed no significant difference between pregnant women with $\mathrm{LN}$ and those without LN. ${ }^{20,22,23}$

Five cases of congenital malformation were found in two included studies. ${ }^{20,23}$ There was no significant difference between pregnant women with $\mathrm{LN}$ and those without $\mathrm{LN}$. Gladman et al reported one infant with cleft lip; one with cleft lip/palate, facial palsy, and visual/hearing impairment; and one with bilateral 2nd and 3rd toe syndactyly. Ku et al reported one neonate with tetralogy of Fallot, and another with atrial septal defect.

There were three studies that reported 1-min Apgar scores. Bramham et al analyzed neonates with 1-min Apgar scores $<7$ and found no difference between the $\mathrm{LN}$ and non-LN groups. ${ }^{24} \mathrm{Lv}$ et al and $\mathrm{Ku}$ et al recorded 1-min Apgar scores (mean \pm standard deviation), although neither study found a significant difference between the two groups. ${ }^{23,25}$ The results are shown in Appendix 5 Table S11B.

\section{Antenatal management}

We analyzed four antenatal management outcomes. Among them, three outcomes were included in the quantitative synthesis, and one outcome was analyzed qualitatively. The outcomes are shown in Appendix 4 Table S10.

\section{Quantitative synthesis and heterogeneity analyses}

In the present study, antenatal management in pregnant women with SLE with LN was compared with antenatal management in pregnant women with SLE without LN. Pregnant 
women with LN presented a significant association with steroid treatment $(\mathrm{OR}=2.48,95 \% \mathrm{CI}=1.59-3.87)$, with statistical heterogeneity across studies $\left(I^{2}=27 \%, P_{Q}=0.24\right)$, and the fixed-effects model was reported. Immunosuppressant treatment was divided into three subgroups according to different outcome definitions (azathioprine, cyclosporin A, and all immunosuppressants), and was significantly associated with pregnant women with SLE with LN, either in subgroups

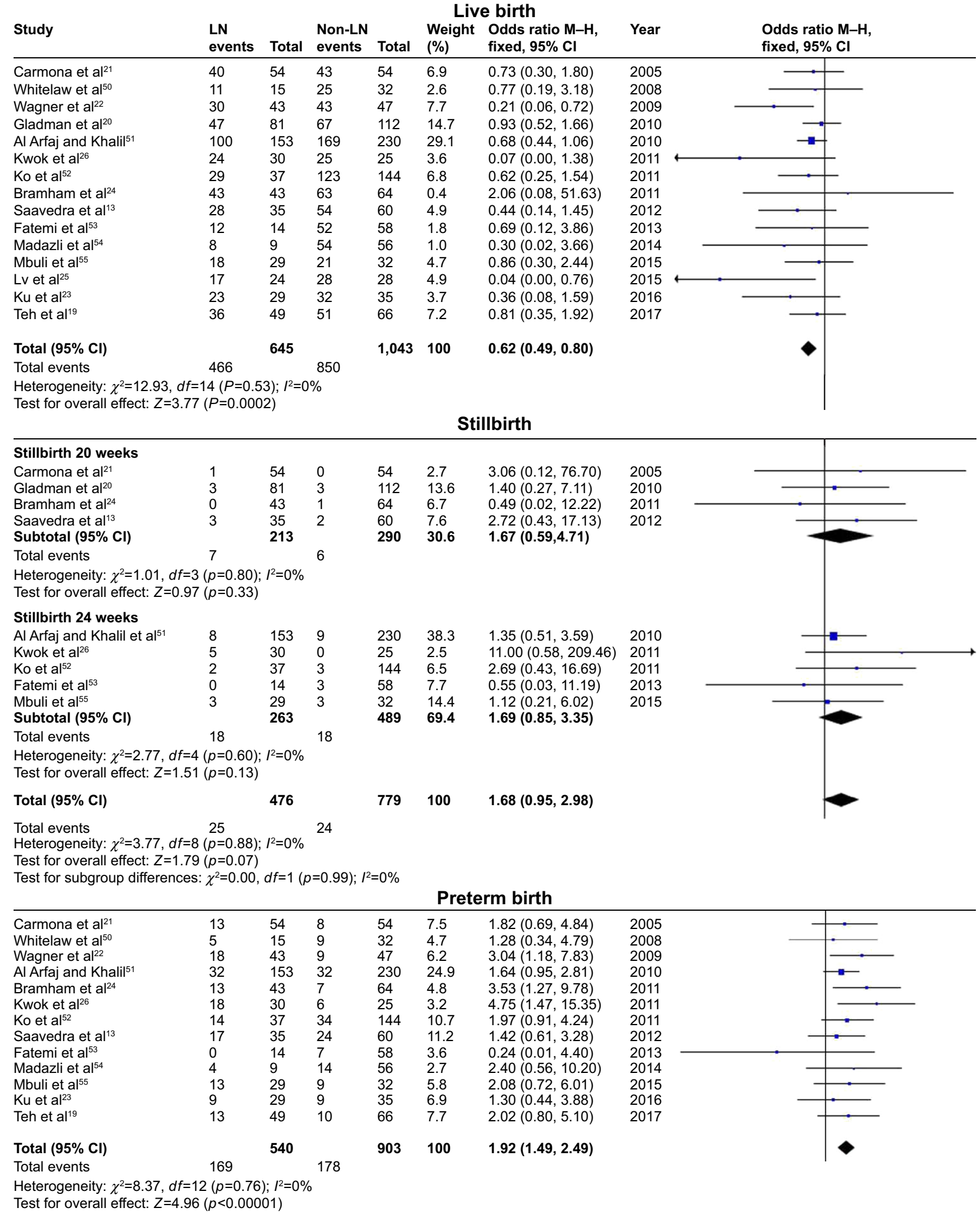

Figure 3 (Continued) 


\begin{tabular}{|c|c|c|c|c|c|c|}
\hline \multirow[b]{2}{*}{ Whitelaw et $a^{50}$} & \multirow[b]{2}{*}{1} & \multirow[b]{2}{*}{15} & \multirow[b]{2}{*}{4} & \multicolumn{3}{|c|}{ LBW/IUGR/SGA } \\
\hline & & & & 32 & 3.1 & $0.50(0.05,4.90)$ \\
\hline Wagner et $\mathrm{al}^{22}$ & 2 & 43 & 2 & 47 & 2.4 & $1.10(0.15,8.15)$ \\
\hline Al Arfaj and Khalil ${ }^{51}$ & 28 & 153 & 32 & 230 & 27.2 & $1.39(0.80,2.41)$ \\
\hline Gladman et $\mathrm{al}^{20}$ & 18 & 81 & 13 & 112 & 11.0 & $2.18(1.00,4.75)$ \\
\hline Kwok et $\mathrm{al}^{26}$ & 14 & 30 & 5 & 25 & 3.8 & $3.50(1.04,11.79)$ \\
\hline Bramham et $\mathrm{al}^{24}$ & 14 & 43 & 14 & 64 & 9.9 & $1.72(0.72,4.12)$ \\
\hline Ko et $\mathrm{al}^{52}$ & 4 & 37 & 20 & 144 & 9.5 & $0.75(0.24,2.35)$ \\
\hline Saavedra et al $^{13}$ & 10 & 35 & 21 & 60 & 14.4 & $0.74(0.30,1.84)$ \\
\hline Lv et $\mathrm{al}^{25}$ & 8 & 24 & 4 & 28 & 3.2 & $3.00(0.77,11.65)$ \\
\hline $\mathrm{Ku}$ et $\mathrm{al}^{23}$ & 9 & 29 & 10 & 35 & 8.1 & $1.13(0.38,3.30)$ \\
\hline Teh et al ${ }^{19}$ & 8 & 49 & 8 & 66 & 7.4 & $1.41(0.49,4.08)$ \\
\hline Total $(95 \% \mathrm{Cl})$ & & 539 & & 843 & 100 & $1.43(1.08,1.91)$ \\
\hline Total events & 116 & & 133 & & & \\
\hline \multicolumn{7}{|c|}{$\begin{array}{l}\text { Heterogeneity: } \chi^{2}=8.84, d f=10(p=0.55) ; l^{2}=0 \% \\
\text { Test for overall effect: } Z=2.46(p=0.01)\end{array}$} \\
\hline
\end{tabular}

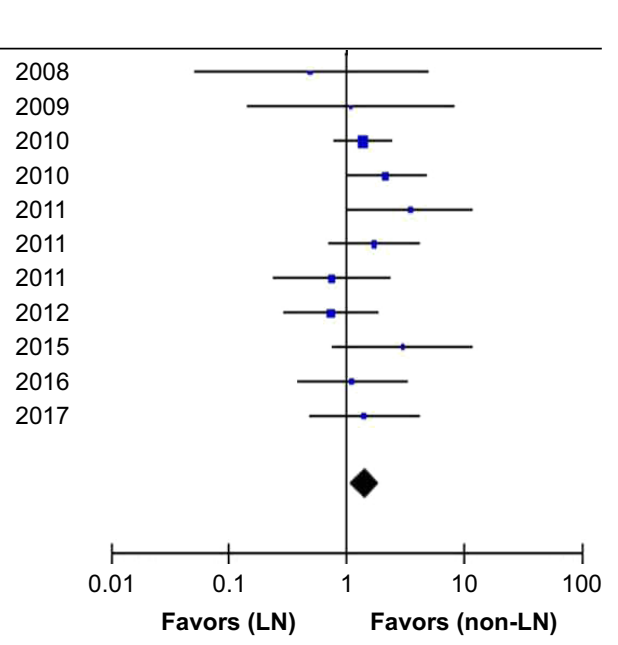

Figure 3 Fetal outcomes observed in pregnant women with SLE with lupus nephritis versus those without lupus nephritis.

Abbreviations: SLE, systemic lupus erythematosus; LN, lupus nephritis; LBW, low birth weight; IUGR, intrauterine growth restriction; SGA, small for gestational age.

or in total $(\mathrm{OR}=6.77,95 \% \mathrm{CI}=3.30-13.89)$, with statistical heterogeneity across studies $\left(I^{2}=53 \%, P_{Q}=0.04\right)$, and the random-effects model was used.

However, antimalarial treatment was not significantly different between pregnant women with $\mathrm{LN}$ and those without LN, with statistical heterogeneity $\left(I^{2}=72 \%, P_{Q}=0.006\right)$, and the random-effects model was used.

The results of antenatal outcomes are shown in Figure 4 and Table 3.

\section{Sensitivity analyses}

Regarding the use of antimalarials, statistical heterogeneity was high $\left(I^{2}=72 \%, P_{Q}=0.006\right)$. Therefore, sensitivity analyses were performed (Table 4B). The sensitivity analyses showed that after omitting the study by Kwok et al, antimalarial treatment was still not significantly different between pregnant women with $\mathrm{LN}$ and those without $\mathrm{LN}$ $\left(\mathrm{OR}=0.89,95 \% \mathrm{CI}=0.54-1.46, I^{2}=28 \%, P_{Q}=0.25\right)$. These data indicated that the pooled result remained robust in the antimalarials outcome.

We also found that the immunosuppressant treatment outcome had high heterogeneity $\left(I^{2}=53 \%, P_{Q}=0.04\right)$, and that the high heterogeneity was mainly from the azathioprine subgroup $\left(I^{2}=71 \%, P_{Q}=0.01\right)$, with the other two subgroups having low heterogeneity (cyclosporin: $P^{2}=0 \%, P_{Q}=0.43$; All immunosuppressants: $P=0 \%, P_{Q}=0.42$ ). Therefore, sensitivity analyses were performed (Table 4C). After omitting the study by Bramham et al, immunosuppressant treatment was still significantly associated with pregnant women with SLE with $\mathrm{LN}$, either in the azathioprine subgroup ( $\mathrm{OR}=4.66$, $\left.95 \% \mathrm{CI}=2.04-10.66, I^{2}=20 \%, P_{Q}=0.28\right)$ or in total $(\mathrm{OR}=4.72$,
95\% CI $\left.=2.84-7.83, I^{2}=0 \%, P_{Q}=0.53\right)$. These data indicated that the pooled results remained robust in the immunosuppressant treatment outcome.

\section{Qualitative analyses}

There were two studies that analyzed the use of aspirin during pregnancy. ${ }^{24,26}$ Bramham et al found that patients with LN were more frequently taking low-dose aspirin compared with patients without LN. However, Kwok et al found no difference between the two groups. The results are shown in Appendix 5 Table S11C.

\section{Risk of bias across studies}

Funnel plots allowed for visualization of the relationship of publication bias among the included studies in this metaanalysis. Figures 5-7 show the funnel plots evaluating the three categories of outcomes (maternal outcomes, fetal outcomes, and antenatal management). In these analyses, the shape of each funnel plot appeared almost symmetrical, meaning that publication bias was mildly evident across all studies.

\section{Discussion}

This systematic review provided a complete overview of published reports on management and pregnancy outcome in women with LN. Overall, we found that LN was associated with higher risk for adverse maternal outcomes such as gestational hypertension, preeclampsia, SLE flare, renal flare, proteinuria during pregnancy, and hypocomplementemia. Furthermore, LN was associated with lower rate of live births, higher rate of preterm births, and significantly higher 


\begin{tabular}{|c|c|c|c|c|c|c|c|c|}
\hline Study & $\begin{array}{l}\text { LN } \\
\text { events }\end{array}$ & Total & $\begin{array}{l}\text { Non-LN } \\
\text { events }\end{array}$ & Total & $\begin{array}{l}\text { Weight } \\
(\%)\end{array}$ & $\begin{array}{l}\text { Steroids } \\
\text { Odds ratio } \mathrm{M}-\mathrm{H} \text {, } \\
\text { fixed, } 95 \% \mathrm{Cl}\end{array}$ & Year & $\begin{array}{l}\text { Odds ratio } \mathrm{M}-\mathrm{H} \text {, } \\
\text { fixed, } 95 \% \mathrm{Cl}\end{array}$ \\
\hline 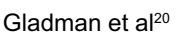 & 61 & 81 & 66 & 112 & 52.5 & $2.13(1.13,3.99)$ & 2010 & - \\
\hline Bramham et $\mathrm{al}^{24}$ & 37 & 43 & 34 & 64 & 14.6 & $5.44(2.02,14.68)$ & 2011 & \\
\hline Ko et al ${ }^{52}$ & 22 & 30 & 15 & 25 & 16.8 & $1.83(0.59,5.72)$ & 2011 & $\longrightarrow$ \\
\hline Saavedra et al ${ }^{13}$ & 32 & 35 & 56 & 60 & 13.6 & $0.76(0.16,3.62)$ & 2012 & $\rightarrow-$ \\
\hline Fatemi et $a^{53}$ & 14 & 14 & 48 & 58 & 2.5 & $6.28(0.35,113.77)$ & 2013 & 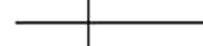 \\
\hline Total $(95 \% \mathrm{Cl})$ & & 203 & & 319 & 100 & $2.48(1.59,3.87)$ & & \\
\hline $\begin{array}{l}\text { Total events } \\
\text { Heterogeneity: } \chi^{2} \\
\text { Test for overall ef }\end{array}$ & $\begin{array}{l}166 \\
51, d f=4 \\
: Z=4.00\end{array}$ & $\begin{array}{l}P=0.24) \\
P<0.000\end{array}$ & $\begin{array}{l}219 \\
l^{2}=27 \% \\
\text { 1) }\end{array}$ & & & & & \\
\hline
\end{tabular}

\section{Antimalarials}

$\mathrm{M}-\mathrm{H}$, random, 95\% $\mathrm{Cl}$ Year $\mathrm{M}-\mathrm{H}$, random, $95 \% \mathrm{Cl}$

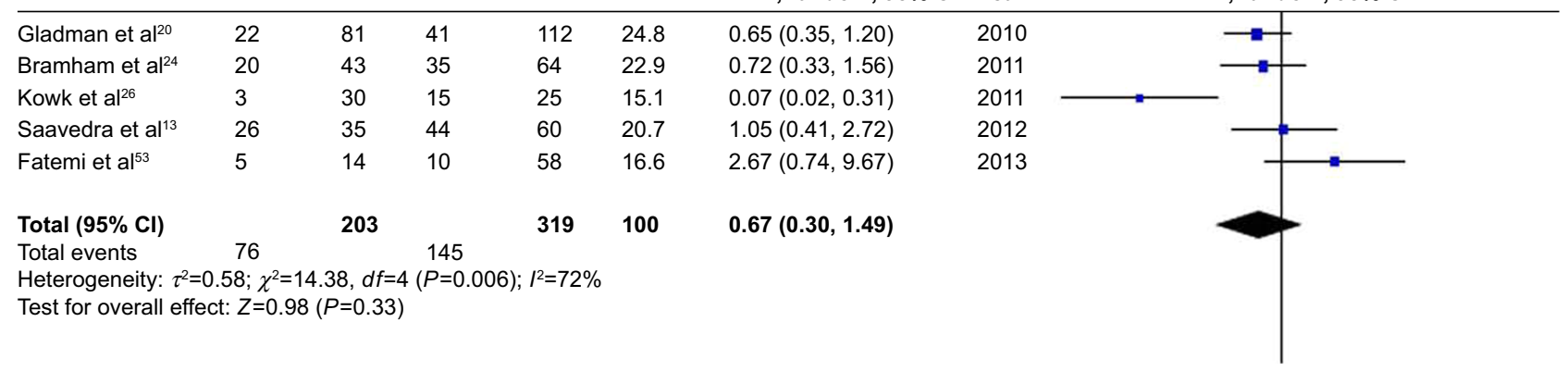

\section{Immunosuppressant}

$\mathrm{M}-\mathrm{H}$, random, $95 \% \mathrm{Cl}$ Year

$\mathrm{M}-\mathrm{H}$, random, $95 \% \mathrm{Cl}$

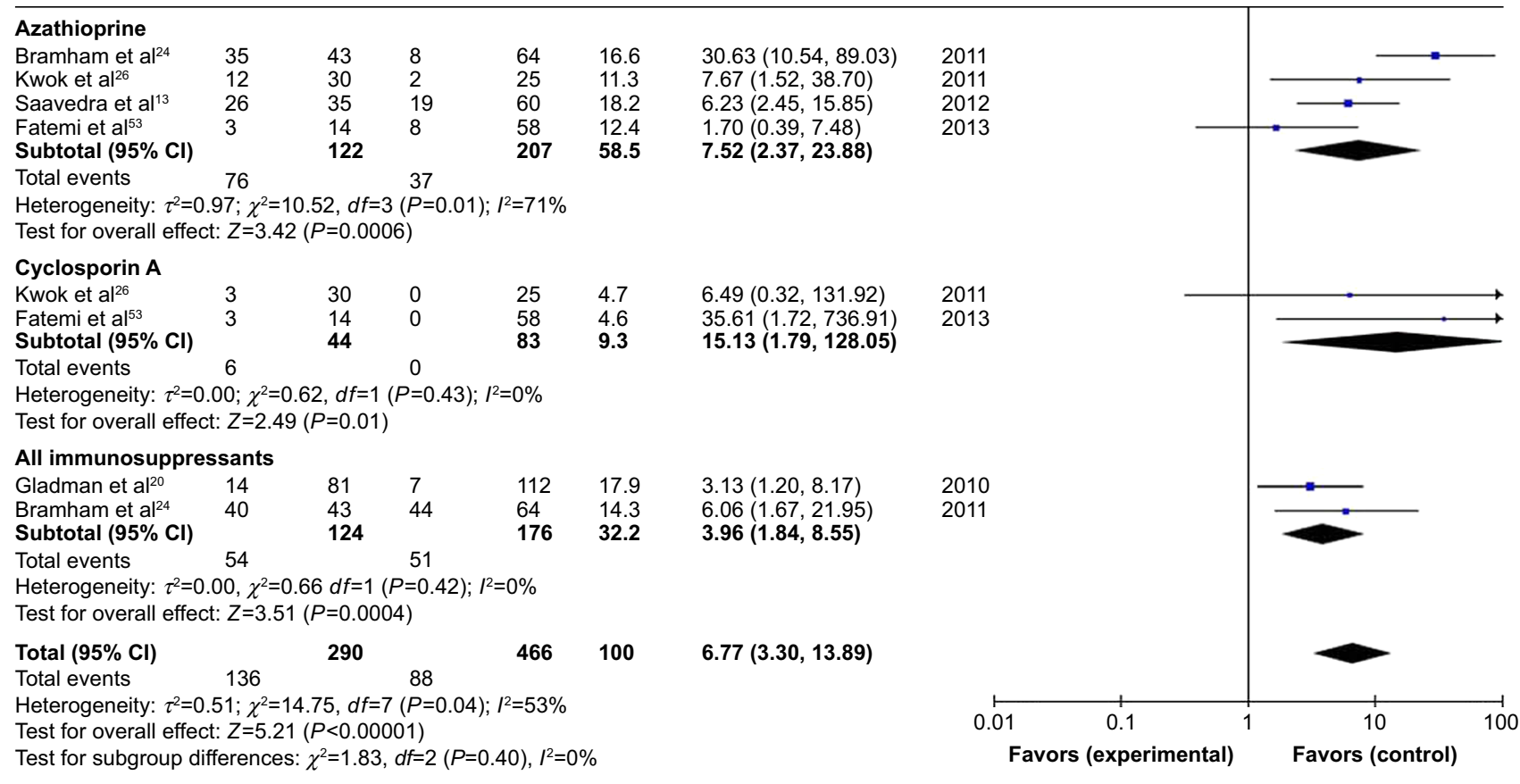

Figure 4 Antenatal management observed in pregnant women with SLE with lupus nephritis versus those without lupus nephritis. Abbreviations: SLE, systemic lupus erythematosus; LN, lupus nephritis.

number of infants with growth restriction (LBW/IUGR/ SGA). There were also significant differences in medications used. For example, women with SLE with LN had increased treatment with steroids and immunosuppressants compared with those without LN.
Recently, a systematic review of women with SLE and the risk of preterm birth showed that preterm birth was significantly associated with patients with SLE with a history of LN, or in patients with active nephritis. ${ }^{27}$ However, the review mainly focused on the association of SLE or 


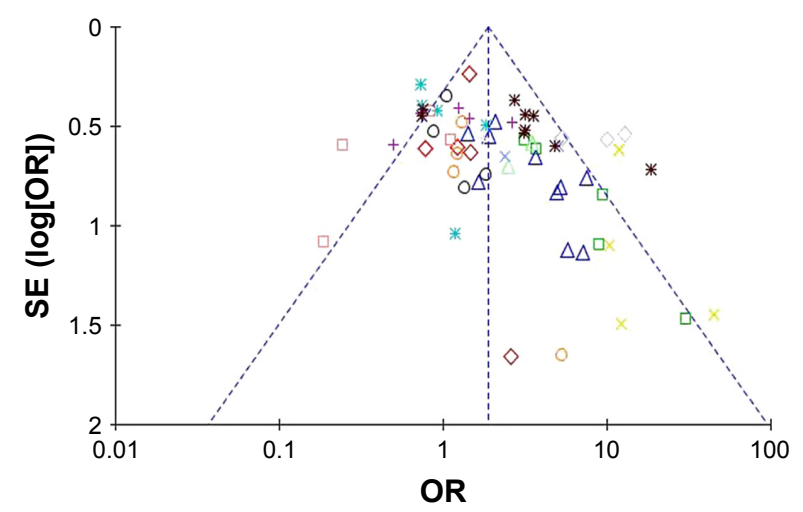

\begin{tabular}{|ll|}
\hline Subgroups & \\
$\bigcirc$ Spontaneous abortion 20 weeks & APS \\
$\diamond$ Spontaneous abortion 24 weeks & Proteinuria \\
$\square$ Gestational hepertension & Anti-SSA/Ro+ \\
$\triangle$ Preeclampsia & Low C3 \\
$\times$ Renal flare & $\times$ Low C4 \\
+ Cesarean section & Low C3 or Low C4 \\
$*$ aPL+ & SLE flare \\
\hline
\end{tabular}

Figure 5 Funnel plot of the association between lupus nephritis and maternal outcomes.

Abbreviations: $\mathrm{SE}$, standard error; OR, odds ratio; aPL+, antiphospholipid antibodies positive; APS, antiphospholipid syndrome; anti-SSA/Ro+, anti-Sjögren's syndromerelated antigen $\mathrm{A} /$ Ro autoantibodies positive; SLE, systemic lupus erythematosus.

LN with preterm birth and did not include other pregnancy outcomes. Similarly, a systemic review of pregnancy outcomes in women with SLE from 2010 showed that LN was associated with premature birth and hypertension during pregnancy, ${ }^{6}$ which was consistent with our analysis. Moreover, the authors indicated the importance of optimal timing of pregnancy in patients with SLE with LN. The present study serves as an updated systemic review, and included a more complete analysis of pregnancy outcomes and antenatal management. Notably, antenatal management was not

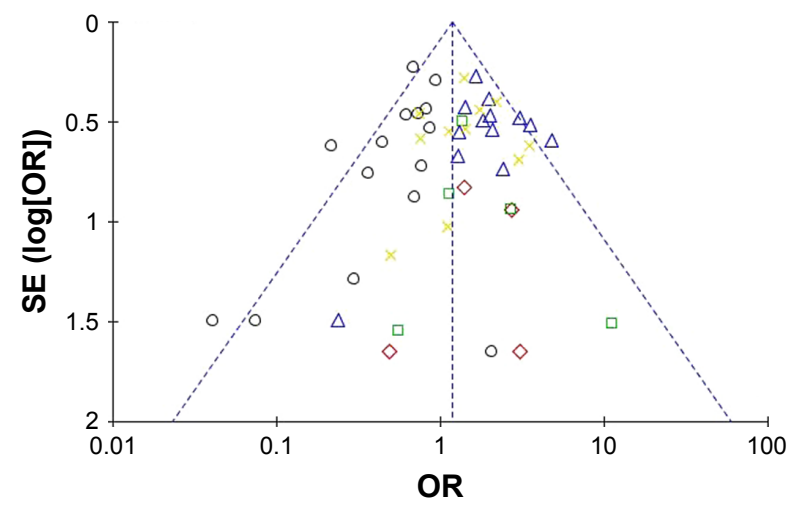

Subgroups
$\bigcirc$ Live birth $\square$ Stillbirth 24 weeks $\times$ LBW/IUGR/SGA
$\diamond$ Stillbirth 20 weeks $\triangle$ Preterm birth

Figure 6 Funnel plot of the association between lupus nephritis and fetal outcomes.

Abbreviations: SE, standard error; OR, odds ratio; LBW, low birth weight; IUGR, intrauterine growth restriction; SGA, small for gestational age.
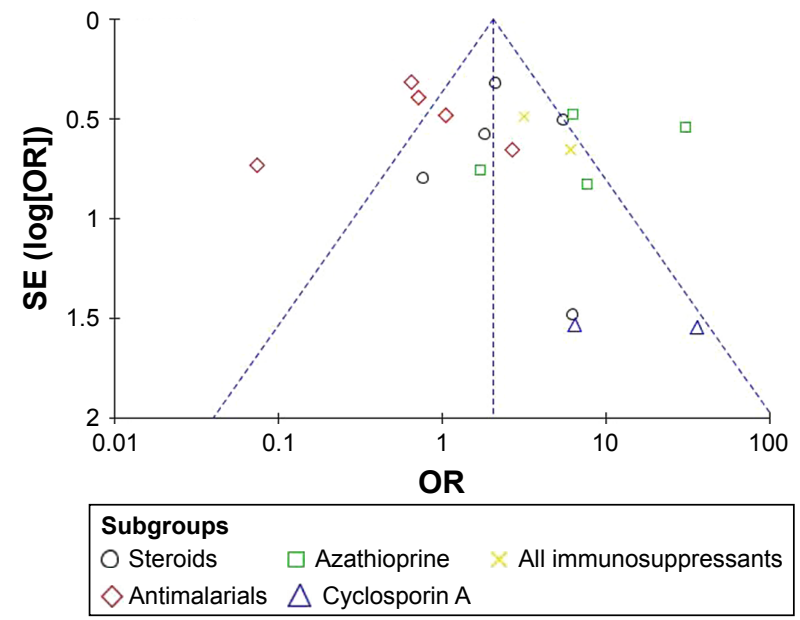

Figure 7 Funnel plot of the association between lupus nephritis and antenatal management.

Abbreviations: SE, standard error; OR, odds ratio.

reported in previous studies. Another meta-analysis showed that SLE had a strong effect on adverse maternal and fetal outcomes following pregnancy, including cesarean section, preeclampsia, hypertension, fetal loss, premature birth, SGA, and congenital defects. ${ }^{28}$ However, the previous metaanalysis reported pregnancy outcomes in women with SLE and those without SLE, and the included studies were limited regarding some of the reported outcomes. In our analysis, we focused on the differences between pregnant women with SLE with LN and those without LN.

The prevalence of SLE is $14.6-50.8$ cases per 100,000 people, ${ }^{29}$ and thus there is a low prevalence of these pregnancy outcomes. Therefore, studies of the associations of LN with pregnancy outcomes are limited, and they have obvious variations in study design, diagnostic criteria, statistical methods, bias, and outcomes analyzed. Furthermore, the study population and status of $\mathrm{LN}$ also varied in these studies. Performing a systemic review, including meta-analyses, allowed for the accumulation of more data for determining the actual associations in the larger population.

$\mathrm{LN}$ was shown to be a strong risk factor for gestational hypertension, preeclampsia, preterm birth, and fetal loss, which was supported by other studies. ${ }^{1,3,4,6}$ One review recommended that for patients with SLE with LN, SLE should be in the remission stage for at least 6 months before conception because adverse pregnancy outcomes are related to active nephritis. ${ }^{5}$ This may be because of compromised microcirculation in the uteroplacental vessels of SLE patients with high blood pressure and LN. Additionally, pregnancy itself contributes partially to renal impairment and subsequently to renal failure in the late stage of pregnancy. ${ }^{30}$ Clinically, 24-h urinary protein is an indicator of renal function. Our 
analysis showed that proteinuria (300 mg/24 h) had high incidence in women with nephritis, which was consistent with previous studies. This suggested that renal involvement and the presence and degree of proteinuria at the time of conception may contribute to adverse pregnancy outcomes. ${ }^{22,31}$ Thus, it is critical to continuously monitor renal function and proteinuria in pregnant patients with LN.

The flare rate in LN remains controversial. In our analysis, the SLE flare rate and renal flare rate were both higher in the LN group. By contrast, a previous study found lower flare rates in the nephritis group compared with all patterns of SLE (however, the sample-size in the study was relatively small). ${ }^{31}$ In a study of 113 pregnancies with LN, there were $30 \%$ with flares. The study also indicated the risk factor of renal flare associated with renal function during pregnancy: patients with either proteinuria $>1 \mathrm{~g} / 24 \mathrm{~h}$ or glomerular filtration rate $<60 \mathrm{~mL} / \mathrm{min} /$ body surface area had a ninefold increased risk of flare. ${ }^{12}$ Clinically, a woman with a history of $\mathrm{LN}$ and/or renal flare during pregnancy is more likely to have flares in future pregnancies. ${ }^{32}$

Anti-Ro/SSA antibodies have previously been shown to be related to miscarriages and loss of pregnancy, ${ }^{33,34}$ although this association has not yet been proven in more recent studies. ${ }^{35,36}$ The main adverse outcomes related to anti-Ro/SSA antibodies are neonatal lupus and congenital heart block. However, in the present analysis, there was a lower percentage of patients who were positive for antiSSA antibodies in the LN group (Figure 2), which has not been reported in other studies. Unfortunately, our analysis included a small number of participants. Therefore, further investigation into the association of LN with neonatal lupus and congenital heart block is warranted.

Complement is important for the development of the placenta and normal fetus. ${ }^{37}$ It is estimated that up to $20 \%$ of first trimester pregnancy losses are associated with hypocomplementemia. ${ }^{38}$ SLE flares are often associated with low $\mathrm{C} 3$ and $\mathrm{C} 4$ levels, ${ }^{39}$ and normal levels of $\mathrm{C} 3$ and $\mathrm{C} 4$ are associated with a reduction in SLE flares. ${ }^{40}$ In the present analysis, we found a significant association between LN and hypocomplementemia, which was consistent with the observation that LN was associated with a higher risk of pregnancy loss and SLE flare.

A growth-restricted neonate has three times the risk on neonatal death compared with one with normal weight. ${ }^{41}$ Adverse consequences of fetal growth restriction include respiratory infection, cardiovascular or metabolic diseases, and neurodevelopmental retardation. ${ }^{37}$ In our review, we merged three definitions of growth restriction as a single fetal outcome (LBW/SGA/IUGR) to clarify the association with fetal growth restriction, because the included studies used them interchangeably. However, it is important to clarify the definitions of the three terms. LBW refers only to infants with birth weight $\leq 2,500 \mathrm{~g}$, regardless of the gestational age and cause. ${ }^{42}$ SGA refers to infants whose weight is less than the lower $10 \%$ limit of the CI of the normal curve for gestation weight. ${ }^{43}$ IUGR refers to processes that can limit the potential for intrauterine growth of the fetus. ${ }^{44}$ SLE has been reported to be associated with poor fetal growth

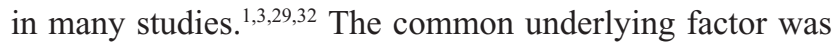
vascular pathology that reduced uteroplacental perfusion. Lupus increases the frequency of fetal growth restriction and preterm birth by $30 \%-50 \%$, which are also affected by the type of nephritis and hypertension. ${ }^{44}$ This was fully demonstrated in our results. These observations indicate that more attention should be paid to fetal growth status in pregnant women with LN.

Immunosuppressive therapy for SLE is often used when there is a flare, or as maintenance therapy for patients in remission. Because most immunosuppressants are teratogenic, azathioprine and cyclosporin are the most commonly used during pregnancy, given that they were proven to be safe for use during pregnancy. ${ }^{45}$ When we compared the use of immunosuppressants and steroids in women with LN and those without LN, we found that women with nephritis tended to have used more medication, which indirectly indicated that the disease was unstable. Antimalarials are now a first-line therapy for SLE in pregnancy. They are safe for pregnancy, and are proven to be associated with a reduced risk of congenital heart block in neonates. ${ }^{46}$ Aspirin has been proven to prevent preeclampsia in women with SLE. ${ }^{47}$ The European League Against Rheumatism and European Renal Association-European Dialysis and Transplant Association guidelines recommend low-dose aspirin therapy for all patients to prevent preeclampsia. ${ }^{48}$ Our analysis included a limited number of studies that involved the use of aspirin, and may represent a direction for further study.

LN as one of the most serious manifestations of SLE apparently increases the risk of both maternal and fetal adverse outcomes. Our data further support the importance of pregnancy planning, pre-conception evaluation, and counseling of women with SLE, especially those with LN. Patients should be informed that disease flare is common during pregnancy, especially in nephritis patients. Therefore, we highly recommend $\mathrm{LN}$ patients to be in remission stage for at least 6 months before conception in order to get positive pregnancy outcome and avoid disease flare. Our study also 
emphasizes the importance of motoring fetal growth status, as fetal growth restriction accounts for one-fifth of the fetuses among nephritis patients. Monitoring strategies should be strengthened in these women during pregnancy.

The present study is noteworthy for several reasons. This is the first systemic review and meta-analysis that comprehensively compared pregnancy outcomes and antenatal management between pregnant women with SLE with and without LN. Additionally, several outcomes were analyzed from data from large sample sizes from different settings. The outcomes were complete and involved almost every key aspect of pregnancy, especially the use of medication, which has not been reported in previous reviews. Furthermore, the present review included the use of NOS and STROBE to evaluate the quality of the included studies, and we performed sensitivity analyses for all results with high heterogeneity.

\section{Limitations}

First, all included studies were retrospective observational studies, in which the results might have reporting bias. Second, a mild level of publication bias existed across studies. Third, there were variations in diagnostic criteria, the measurement of SLE activity, and the percentage of patients who underwent renal biopsy among studies.

\section{Conclusion}

Patients with LN tend to have an increased risk for gestational hypertension, preeclampsia, SLE flare, renal flare, proteinuria, and hypocomplementemia during pregnancy, compared with those without LN. Additionally, LN was shown to contribute to increased incidence of fetal loss, premature birth, and fetal growth restriction. Increased use of steroids and immunosuppressants was found in pregnant women with LN. Therefore, pre-pregnancy counseling, optimal timing of conception, and close monitoring in antenatal care, especially of renal function and blood pressure, are critical in SLE patients with LN. Further studies should be conducted on antenatal management, including assessment and medication use in pregnant women with LN.

\section{Acknowledgment}

The work was supported by funding from the Shanghai Municipal Health and Family Planning Commission (15GWZK0701).

\section{Author contributions}

All authors contributed toward data analysis, drafting and critically revising the paper and agree to be accountable for all aspects of the work.

\section{Disclosure}

The authors report no conflicts of interest in this work.

\section{References}

1. Meyer O. Making pregnancy safer for patients with lupus. Joint Bone Spine. 2004;71(3):178-182.

2. Chen S, Sun X, Wu B, Lian X. Pregnancy in women with systemic lupus erythematosus: a retrospective study of 83 pregnancies at a single centre. Int J Environ Res Public Health. 2015;12(8):9876-9888.

3. Lateef A, Petri M. Systemic lupus erythematosus and pregnancy. Rheum Dis Clin North Am. 2017;43(2):215-226.

4. Khamashta MA. Systemic lupus erythematosus and pregnancy. Best Pract Res Clin Rheumatol. 2006;20(4):685-694.

5. Stanhope TJ, White WM, Moder KG, Smyth A, Garovic VD. Obstetric nephrology: lupus and lupus nephritis in pregnancy. Clin J Am Soc Nephrol. 2012;7(12):2089-2099.

6. Smyth A, Oliveira GH, Lahr BD, Bailey KR, Norby SM, Garovic VD. A systematic review and meta-analysis of pregnancy outcomes in patients with systemic lupus erythematosus and lupus nephritis. Clin $J$ Am Soc Nephrol. 2010;5(11):2060-2068.

7. Liberati A, Altman DG, Tetzlaff J, et al. The PRISMA statement for reporting systematic reviews and meta-analyses of studies that evaluate healthcare interventions: explanation and elaboration. BMJ. 2009; 339:b2700.

8. Gladman DD, Ibañez D, Urowitz MB. Systemic lupus erythematosus disease activity index 2000. J Rheumatol. 2002;29(2):288-291.

9. Bombardier C, Gladman DD, Urowitz MB, Caron D, Chang CH. Derivation of the SLEDAI. A disease activity index for lupus patients. The Committee on Prognosis Studies in SLE. Arthritis Rheum. 1992;35(6): 630-640.

10. Buyon JP, Kalunian KC, Ramsey-Goldman R, et al. Assessing disease activity in SLE patients during pregnancy. Lupus. 1999;8(8): 677-684.

11. Harrington JT. The uses of disease activity scoring and the physician global assessment of disease activity for managing rheumatoid arthritis in rheumatology practice. J Rheumatol. 2009;36(5):925-929.

12. Imbasciati E, Tincani A, Gregorini G, et al. Pregnancy in women with pre-existing lupus nephritis: predictors of fetal and maternal outcome. Nephrol Dial Transplant. 2009;24(2):519-525.

13. Saavedra MA, Cruz-Reyes C, Vera-Lastra O, et al. Impact of previous lupus nephritis on maternal and fetal outcomes during pregnancy. Clin Rheumatol. 2012;31(5):813-819.

14. Wilson WA, Gharavi AE, Koike T, et al. International consensus statement on preliminary classification criteria for definite antiphospholipid syndrome: report of an international workshop. Arthritis Rheum. 1999; 42(7):1309-1311.

15. Busby J, Purdy S, Hollingworth W. A systematic review of the magnitude and cause of geographic variation in unplanned hospital admission rates and length of stay for ambulatory care sensitive conditions. BMC Health Serv Res. 2015;15:324.

16. Ajmani GS, Suh HH, Wroblewski KE, Pinto JM. Smoking and olfactory dysfunction: a systematic literature review and meta-analysis. Laryngoscope. 2017;127(8):1753-1761.

17. Bhindi B, Wallis CJD, Nayan M, et al. The association between vasectomy and prostate cancer: a systematic review and meta-analysis. JAMA Intern Med. 2017;177(9):1273-1286.

18. Vandenbroucke JP, von Elm E, Altman DG, et al; STROBE Initiative. Strengthening the Reporting of Observational Studies in Epidemiology (STROBE): explanation and elaboration. PLoS Med. 2007; 4(10):e297.

19. Teh CL, Wan SA, Cheong YK, Ling GR. Systemic lupus erythematosus pregnancies: ten-year data from a single centre in Malaysia. Lupus. 2017;26(2):218-223.

20. Gladman DD, Tandon A, Ibañez D, Urowitz MB. The effect of lupus nephritis on pregnancy outcome and fetal and maternal complications. J Rheumatol. 2010;37(4):754-758. 
21. Carmona F, Font J, Moga I, et al. Class III-IV proliferative lupus nephritis and pregnancy: a study of 42 cases. Am J Reprod Immunol. 2005; 53(4):182-188.

22. Wagner SJ, Craici I, Reed D, et al. Maternal and foetal outcomes in pregnant patients with active lupus nephritis. Lupus. 2009;18(4):342-347.

23. Ku M, Guo S, Shang W, et al. Pregnancy outcomes in Chinese patients with systemic lupus erythematosus (SLE): a retrospective study of 109 pregnancies. PLoS One. 2016;11(7):e0159364.

24. Bramham K, Hunt BJ, Bewley S, et al. Pregnancy outcomes in systemic lupus erythematosus with and without previous nephritis. J Rheumatol. 2011;38(9):1906-1913.

25. Lv J, Wang W, Li Y. Clinical outcomes and predictors of fetal and maternal consequences of pregnancy in lupus nephritis patients. Int Urol Nephrol. 2015;47(8):1379-1385.

26. Kwok LW, Tam LS, Zhu T, Leung YY, Li E. Predictors of maternal and fetal outcomes in pregnancies of patients with systemic lupus erythematosus. Lupus. 2011;20(8):829-836.

27. Wei S, Lai K, Yang Z, Zeng K. Systemic lupus erythematosus and risk of preterm birth: a systematic review and meta-analysis of observational studies. Lupus. 2017;26(6):563-571.

28. Bundhun PK, Soogund MZ, Huang F. Impact of systemic lupus erythematosus on maternal and fetal outcomes following pregnancy: a meta-analysis of studies published between years 2001-2016. J Autoimmun. 2017;79:17-27.

29. Singh AG, Chowdhary VR. Pregnancy-related issues in women with systemic lupus erythematosus. Int J Rheum Dis. 2015;18(2):172-181.

30. Koh JH, Ko HS, Lee J, et al. Pregnancy and patients with preexisting lupus nephritis: 15 years of experience at a single center in Korea. Lupus. 2015;24(7):764-772.

31. Huong DL, Wechsler B, Vauthier-Brouzes D, Beaufils H, Lefebvre G, Piette JC. Pregnancy in past or present lupus nephritis: a study of 32 pregnancies from a single centre. Ann Rheum Dis. 2001;60(6):599-604.

32. Clowse ME. Lupus activity in pregnancy. Rheum Dis Clin North Am. 2007;33(2):237-252, v.

33. Hull RG, Harris EN, Morgan SH, Hughes GR. Anti-Ro antibodies and abortions in women with SLE. Lancet. 1983;2(8359):1138.

34. Watson RM, Braunstein BL, Watson AJ, Hochberg MC, Provost TT Fetal wastage in women with anti-Ro(SSA) antibody. J Rheumatol. 1986;13(1):90-94.

35. Brucato A, Doria A, Frassi M, et al. Pregnancy outcome in 100 women with autoimmune diseases and anti-Ro/SSA antibodies: a prospective controlled study. Lupus. 2002;11(11):716-721.

36. Martínez-Sánchez N, Pérez-Pinto S, Robles-Marhuenda Á, et al. Obstetric and perinatal outcome in anti-Ro/SSA-positive pregnant women: a prospective cohort study. Immunol Res. 2017;65(2):487-494.

37. Regal JF, Gilbert JS, Burwick RM. The complement system and adverse pregnancy outcomes. Mol Immunol. 2015;67(1):56-70.

38. Cunningham DS, Tichenor JR Jr. Decay-accelerating factor protects human trophoblast from complement-mediated attack. Clin Immunol Immunopathol. 1995;74(2):156-161.

39. Birmingham DJ, Irshaid F, Nagaraja $\mathrm{HN}$, et al. The complex nature of serum C3 and C4 as biomarkers of lupus renal flare. Lupus. 2010;19(11): 1272-1280.

40. Stohl W, Hiepe F, Latinis KM, et al; BLISS-52 Study Group; BLISS-76 Study Group. Belimumab reduces autoantibodies, normalizes low complement levels, and reduces select B cell populations in patients with systemic lupus erythematosus. Arthritis Rheum. 2012;64(7):2328-2337.
41. Garite TJ, Clark R, Thorp JA. Intrauterine growth restriction increases morbidity and mortality among premature neonates. Am J Obstet Gynecol. 2004;191(2):481-487.

42. WHO. Aspects of low birth weight. Report of the expert committee of maternal child health. WHO Tech Rep. 1961;217:3-16.

43. Goto E. Maternal anthropometry to predict small for gestational age: a meta-analysis. Eur J Obstet Gynecol Reprod Biol. 2016;203:193-198.

44. Valero De Bernabé J, Soriano T, Albaladejo R, et al. Risk factors for low birth weight: a review. Eur J Obstet Gynecol Reprod Biol. 2004;116(1):3-15.

45. Kattah AG, Garovic VD. Pregnancy and lupus nephritis. Semin Nephrol. 2015;35(5):487-499.

46. Izmirly PM, Costedoat-Chalumeau N, Pisoni CN, et al. Maternal use of hydroxychloroquine is associated with a reduced risk of recurrent anti-SSA/Ro-antibody-associated cardiac manifestations of neonatal lupus. Circulation. 2012;126(1):76-82.

47. Schramm AM, Clowse ME. Aspirin for prevention of preeclampsia in lupus pregnancy. Autoimmune Dis. 2014;2014:920467.

48. Bertsias GK, Tektonidou M, Amoura Z, et al; European League Against Rheumatism and European Renal Association-European Dialysis and Transplant Association. Joint European League Against Rheumatism and European Renal Association-European Dialysis and Transplant Association (EULAR/ERA-EDTA) recommendations for the management of adult and paediatric lupus nephritis. Ann Rheum Dis. 2012;71(11):1771-1782.

49. Cavallasca JA, Laborde HA, Ruda-Vega H, Nasswetter GG. Maternal and fetal outcomes of 72 pregnancies in Argentine patients with systemic lupus erythematosus (SLE). Clin Rheumatol. 2008;27(1):41-46.

50. Whitelaw DA, Hall D, Kotze T. Pregnancy in systemic lupus erythematosus: a retrospective study from a developing community. Clin Rheumatol. 2008;27(5):577-580.

51. Al Arfaj AS, Khalil N. Pregnancy outcome in 396 pregnancies in patients with SLE in Saudi Arabia. Lupus. 2010;19(14):1665-1673.

52. Ko HS, Ahn HY, Jang DG, et al. Pregnancy outcomes and appropriate timing of pregnancy in 183 pregnancies in Korean patients with SLE. Int J Med Sci. 2011;8(7):577-583.

53. Fatemi A, Fard RM, Sayedbonakdar Z, Farajzadegan Z, Saber M. The role of lupus nephritis in development of adverse maternal and fetal outcomes during pregnancy. Int J Prev Med. 2013;4(9):1004-1010.

54. Madazli R, Yuksel MA, Oncul M, Imamoglu M, Yilmaz H. Obstetric outcomes and prognostic factors of lupus pregnancies. Arch Gynecol Obstet. 2014;289(1):49-53.

55. Mbuli L, Mapiye D, Okpechi I. Lupus nephritis is associated with poor pregnancy outcomes in pregnant SLE patients in Cape Town: a retrospective analysis. Pan Afr Med J. 2015;22:365.

56. Hochberg MC. Updating the American College of Rheumatology revised criteria for the classification of systemic lupus erythematosus. Arthritis Rheum. 1997;40(9):1725.

57. Tan EM, Cohen AS, Fries JF, et al. The 1982 revised criteria for the classification of systemic lupus erythematosus. Arthritis Rheum. 1982 . 25(11):1271-1277.

58. Churg J, Bernstein J, Glassock RJ. Renal Diseases: Classification and Atlas of Glomerular Diseases. 2nd ed. New York: Igaku-Shoin; 1995.

59. Weening JJ, D’Agati VD, Schwartz MM, et al; International Society of Nephrology Working Group on the Classification of Lupus Nephritis; Renal Pathology Society Working Group on the Classification of Lupus Nephritis. The classification of glomerulonephritis in systemic lupus erythematosus revisited. Kidney Int. 2004;65(2):521-530.
Therapeutics and Clinical Risk Management

\section{Publish your work in this journal}

Therapeutics and Clinical Risk Management is an international, peerreviewed journal of clinical therapeutics and risk management, focusing on concise rapid reporting of clinical studies in all therapeutic areas, outcomes, safety, and programs for the effective, safe, and sustained use of medicines. This journal is indexed on PubMed Central, CAS,

\section{Dovepress}

EMBase, Scopus and the Elsevier Bibliographic databases. The manuscript management system is completely online and includes a very quick and fair peer-review system, which is all easy to use. Visit http://www.dovepress.com/testimonials.php to read real quotes from published authors. 